\title{
Grenzen. Räumliche und soziale Trennlinien im Zeitverlauf
}

\author{
Andrea Komlosy \\ Wien: Promedia 2018, 247 Seiten.
}

\section{Dieter Segert}

Institut für Politikwissenschaft, Universität Wien, Austria

E-Mail: dieter.segert@univie.ac.at
In den jüngsten Wahlkämpfen in Italien, Österreich und Deutschland spielten Forderungen nach einer Sicherung der Außengrenzen eine zentrale Rolle. Doch sind die Grenzen überhaupt jemals ungesichert gewesen? Und lassen sie sich eigentlich vollständig für Fremde schließen? In Zeiten einer großen Verunsicherung über die eigene Zukunft haben Mythen Konjunktur. Da ist es gut, wenn sich das Buch der Historikerin Andrea Komlosy um Klarheit in der Begrifflichkeit und der Geschichte von Grenzen bemüht. Die Autorin hat sich bereits seit vielen Jahren wissenschaftlich mit Grenzen beschäftigt und ist als Globalhistorikerin mit einer soliden wirtschaftswissenschaftlichen Kompetenz für eine Behandlung des Themas bestens geeignet.

Natürlich bedarf ein umgangssprachlicher Begriff wie "Grenze“ zunächst einer genauen begriffsgeschichtlichen Einordnung. Das wird in einem besonderen $\mathrm{Ab}-$ schnitt erledigt, an dessen Ende sich eine informative tabellarische Übersicht seiner Bedeutungen in verschiedenen Sprachen findet (I6). Bezüglich der aktuellen politischen Debatte über die "Grenzen“ konfrontiert die Autorin uns mit ihrer Position, dass weder die Gruppe, die nach "no border" ruft, noch die Gruppe, welche die unverzügliche Schließung der Grenzen fordert, eine verallgemeinerbare Politik vorschlägt. Ihre These ergibt sich aus der Frage danach, welche sozialen Interessen hinter den jeweiligen Extremen stehen, und ihre Antwort, dass beide Positionen einseitig sind (8), ist überzeugend. Insofern ist die mit dem Buch vorgenommene wissenschaftliche Erörterung der den jeweiligen Vorschlägen zur Nutzung von Grenzen zugrunde liegenden „Machtverhältnissen, Aushandlungssystemen, gesellschaftlichen Interessen und Entwürfen" eine Voraussetzung dafür, Mythen und Einseitigkeiten zu vermeiden.
Politikwissenschaftliche Themen, wie es die der Grenzen (und den damit zusammenhängenden Herrschaftsverhältnissen) sind, gewinnen durch eine historische Analyse. Das ist die erste Schlussfolgerung des Rezensenten beim Lesen des Buches. Es vermindert die Blindheit spezialisierter Fachdebatten, wenn wir über den Tellerrand der eigenen Disziplin zu blicken vermögen und wie in diesem Buch von der Geschichtswissenschaft lernen. Dem ersten und dritten Kapitel liegt eine chronologische Darstellung des jeweiligen Gegenstandes zugrunde, aus der dann deutlich wird, dass Grenzen sich in ihrer Gestalt und auch ihrer Wahrnehmung in der jeweiligen Zeit deutlich verändern. Allein schon diese historischen Skizzen (über die Entstehung von Staatlichkeit, deren besondere Form im westeuropäischen Feudalismus, die Auseinandersetzungen des I9. Jahrhunderts über Protektionismus und Freihandel) machen das Buch zu einer anregenden Lektüre für einen Politikwissenschaftler. Dabei steht die Analyse unserer heutigen Epoche, die mit den I980er Jahren einsetzt und nach der Wirtschaftskrise von 2008 eine erneute Dynamisierung erfährt, im Zentrum des Interesses der Autorin.

Im zweiten Kapitel werden verschiedene Formen der Grenze im Überblick behandelt, wobei eine Besonderheit dieser Abhandlung sicher darin besteht, dass neben vielfältigen Grenzen im Bereich der Politik (u.a. Militärgrenzen, Frontiers) auch solche der Kultur und der sozialen Beziehungen, deren Gestalt und Durchlässigkeit, behandelt werden. Der Begriff einer "Phantomgrenze“" ist zunächst rätselhaft, wird jedoch genau erklärt: alte Abgrenzungen können in Phasen der Krise eines politischen Gemeinwesens wieder ins Zentrum der öffentlichen Auseinandersetzung aufsteigen. Ob aber jene aus dem Nebel der Geschichte wiederaufgestiegenen Sonde- 
ridentitäten (wie die Galizische in der Ukraine) wirksam werden, entscheidet sich erst in aktuellen politischen Auseinandersetzungen (I29-I30).

Das dritte Kapitel stellt eine systematische Abhandlung unterschiedlicher Grenzregime dar, des Warenund Kapitalverkehrs, des Personenverkehrs, sozialer Auf- und Abstiege und kultureller Grenzen. Besonders interessant ist der dichte Text zur Migration, der die unterschiedlichen Interessenlagen und Gelegenheiten zur Aus- bzw. Einwanderung darstellt. Komlosy macht deutlich, dass die gegenwärtige Politik der reichen europäischen Staaten in einer alten Traditionslinie zur Absicherung ihrer wirtschaftlichen Überlegenheit steht. Wie jene „Festung Europa“ durch Grenzregime gesichert wird, und welche Folgen das für unsere Gesellschaften wie auch für die ImmigrantInnen hat, wird detailliert erläutert.

Die Autorin versteht es, die einer Politik zugrundeliegenden Interessenkämpfe sichtbar zu machen. Und so ist es nicht verwunderlich, dass sie auch die Interessen des globalen Südens zur Sprache bringt. „Die Auseinandersetzung mit Grenzen ist [...] auch eine Methode zum Erkennen globaler Ungleichheit, die nach dem Ende der Kolonialära in der Schieflage der internationalen Beziehungen fortlebt." (232) 